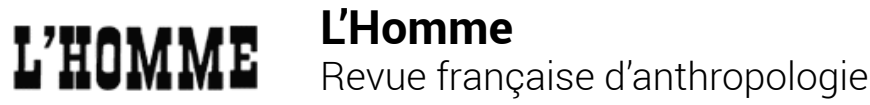

229 | 2019

Varia

\section{Discussion autour de la notion de patriarcat, en Chine et en anthropologie}

\section{Catherine Capdeville-Zeng}

\section{(2) OpenEdition \\ 1 Journals}

Édition électronique

URL : https://journals.openedition.org//homme/33271

DOI : 10.4000//homme.33271

ISSN : 1953-8103

Éditeur

Éditions de l'EHESS

\section{Édition imprimée}

Date de publication : 1 mars 2019

Pagination : 99-134

ISSN : 0439-4216

\section{Référence électronique}

Catherine Capdeville-Zeng, « Discussion autour de la notion de patriarcat, en Chine et en

anthropologie », L'Homme [En ligne], 229 | 2019, mis en ligne le 01 mars 2019, consulté le 08 janvier 2022. URL : http://journals.openedition.org//homme/33271 ; DOI : https://doi.org/10.4000//homme 33271

(c) École des hautes études en sciences sociales 



\title{
Discussion autour de la notion de patriarcat, en Chine et en anthropologie
}

\author{
Catherine Capdeville-Zeng
}

$\mathrm{L}_{\mathrm{s}}$ particulièrement anglo-saxonne, dès lors qu'il s'agit de parler de la société chinoise des Han et de son organisation familiale. Le livre dont il est question ici reprend ce mot dans son titre Le Patriarcat en transformation. Les familles chinoises au XXI siècle ${ }^{1}$, et les articles qui le composent l'utilisent tous, quoique dans des acceptions parfois différentes. Cet ouvrage fait suite à un colloque organisé en juin 2013 par le Max Planck Institute for Social Anthropology (Halle, Allemagne) sur le thème "Is Chinese Patriarchy Over?». L'importance de cette notion de patriarcat, que l'on retrouve chez ces scientifiques donc, mais aussi actuellement dans les articles de journaux et de revues qui la convoquent largement pour rapporter et dénoncer les pratiques de harcèlement sexuel envers les femmes depuis l'affaire Harvey Weinstein, ou encore dans la bouche de nombreux étudiants de mes cours d'anthropologie de la Chine qui l'emploient très fréquemment, interroge. Toutes formes de hiérarchie et de discrimination envers les femmes en viennent aujourd'hui à être qualifiées de "patriarcales", et la Chine incarnerait l'image d'une société profondément patriarcale.

Je m’intéresserai d'abord ici aux acceptions du mot "patriarcat» proposées par les coordinateurs de l'ouvrage dans leur introduction, Stevan Harrell, professeur d'anthropologie à l'Université de Washington, et Gonçalo Santos, professeur assistant à l'Université de Hong Kong, puis j'engagerai une discussion sur l'application de ce terme à la société chinoise. Ensuite, dans

À propos de Gonçalo D. Santos \& Stevan Harrell, eds, Transforming Patriarchy. Chinese Families in the Twenty-First Century, Seattle, University of Washington Press, 2017.

1. Ce titre pourrait aussi se traduire par «La transformation du patriarcat», ou encore "Transformer le patriarcat». N'étant pas angliciste, je laisse là cette discussion. 
une deuxième partie, je présenterai les différents chapitres du livre, issus d'enquêtes de terrain passionnantes menées en Chine et de grande qualité pour la plupart d'entre elles. Dans une troisième partie, je les commenterai à partir de mon expérience personnelle de sinologue et anthropologue. Enfin, je conclurai par une réflexion sur les tendances d'évolution de la société chinoise en matière d'organisation familiale. Tout au long de ces pages, je questionnerai l'adéquation du mot patriarcat et de son emploi unilatéral pour qualifier la Chine.

\section{La notion de patriarcat dans l'ouvrage et sa discussion}

Le patriarcat est d'abord étudié à l'aune de ce qui en est dit dans l'introduction de l'ouvrage, puis de mes connaissances sur la société chinoise. La première section s'appuie entièrement sur les réflexions et la démonstration des auteurs, les extraits traduits ${ }^{2}$ sont toujours explicitement référencés, cependant certains passages sont parfois reformulés pour les rendre plus accessibles en français, ils sont alors présentés sans guillemets. La seconde section fait appel à des sources extérieures à l'ouvrage pour commenter la notion de patriarcat appliquée à la Chine.

\section{Du mot "patriarcat" dans l'introduction de l'ouvrage}

Dès les premières pages de l'introduction, sont évoqués les «arrangements patriarcaux antérieurs» (p. 3) composés de «hiérarchies traditionnelles» (id.) qui caractérisent "un modèle de patriarcat» (p. 4), celui-ci étant défini comme incluant deux axes d'inégalité - génération et genre -, interagissant entre eux et avec l'environnement (id.). Née d'observations empiriques montrant le rapide changement des processus familiaux en Chine contemporaine, tant dans les réalités politiques et économiques qu'aux niveaux du sens et des valeurs, l'idée s'est donc imposée aux co-auteurs de l'ouvrage de dépeindre et de comprendre ces transformations, tout en se démarquant de deux travaux récents qui ont fait parler d'eux: d'abord, celui de l'anthropologue sino-américain Yan Yunxiang (2009a) concernant l'«individualisation » de la société chinoise, parce qu'il aurait le défaut de "surestimer le degré par rapport auquel les individus se sont distancés de la famille ainsi que d'autres liens sociaux et culturels» (p. 6); ensuite, celui dirigé par Deborah Davis et Sara Friedman (2014) sur la «désinstitutionnalisation des relations de familles et de genre", qui souffrirait d'une contradiction due à la valeur toujours affirmée de la famille dans la société chinoise contemporaine (id.).

2. Tous les extraits tirés de cet ouvrage, ou d'autres livres en anglais, ainsi que les titres des contributions examinées ont été traduits par mes soins. 
La situation chinoise est en effet complexe: d'un côté, on assiste à de nouvelles formes de relations familiales (cohabitation avant le mariage, fiancées enceintes, manifestations publiques d'affection, familles moins étendues) qui «suggèrent l'affaiblissement des structures institutionnelles et leur remplacement par des stratégies individuelles» (id.), mais, de l'autre, «les normes et procédures patriarcales antérieures continuent de prévaloir (mariage virilocal, responsabilité des tâches ménagères revenant aux femmes, termes de parenté patricentriques)» (pp. 6-7). On observe, de plus, plusieurs «paradoxes» (p. 7):

«[...] le maintien du devoir de piété filiale se conjugue avec le fait que les belles-filles ont tendance à se substituer aux filles pour prodiguer des soins aux aînés, l'importance croissante accordée à la valeur des filles à l'époque de la politique du planning familial semble contradictoire avec le déséquilibre du sex-ratio en faveur des garçons dans beaucoup de régions rurales, le développement d'une culture des pratiques de cour dans les zones urbaines coexiste avec le pouvoir persistant des anciennes générations d'influencer les décisions de leurs enfants adultes quand il s'agit de choix matrimonial» (id.).

Au vu de ces paradoxes, la question de savoir si le patriarcat perdure s'est transformée en une analyse des reconfigurations des formes de patriarcat entre les périodes antérieures et «l'âge de l'intégration économique et de la globalisation digitale» (id.).

C'est l'histoire du concept de patriarcat et de son application à la société chinoise qui est ensuite abordée. Deux acceptions du terme définies par le Oxford English Dictionary, dont les auteurs pensent qu'elles peuvent s'appliquer à la Chine, sont d'emblée avancées. Selon la première, le patriarcat est «une forme d'organisation sociale dans laquelle le père ou l'homme le plus âgé est le chef de la famille, et où la descendance se fait en ligne agnatique; [une forme de] gouvernement ou [de] domination par un ou des hommes", une définition qui fut employée en anthropologie depuis Lewis Henry Morgan (1877), en passant par Friedrich Engels (1884) et jusque dans les années 1970 (p. 7). Il est mentionné que «la définition de Morgan convient remarquablement pour la famille chinoise de la fin de l'empire et de la période républicaine» (p. 8), dans la mesure où les caractéristiques principales de la famille chinoise patriarcale sont:

«[...] la descendance et l'héritage patrilinéaire, la résidence patrilocale, l'autorité parentale forte et le pouvoir de la génération supérieure (particulièrement, mais pas exclusivement de la part des hommes âgés) renforcé par la loi de l'État et de la propriété des biens» (id.).

Cette idée va ensuite être reprise et développée dans la notion de Patriarchalismus de Max Weber (1978 [1921]), qu'il a décrite comme «une structure de domination dans laquelle le contrôle exercé par l'homme chef de famille n'avait pas d'autres limites que celles fixées par la tradition » (id.), et comme ressemblant à une entreprise qui offre un modèle de relations de 
pouvoir pouvant être étendu à l'extérieur du foyer domestique. Bien que Weber se soit inspiré de la Rome ancienne pour bâtir ce modèle, il peut être utilisé pour la Chine impériale qui s'en rapproche par les traits suivants:

«[...] le contrôle des biens de production par la génération supérieure, la résidence matrimoniale patrilocale, des règles strictes de piété filiale et de respect envers les générations plus âgées, et une idéologie de la supériorité masculine prônant que les plus âgés prévalent sur les jeunes et les hommes sur les femmes» (id.).

Dans les années 1970, une deuxième acception de la définition du Oxford English Dictionary du patriarcat s'impose, influencée par les féministes, qui s'y réferent de façon globale pour désigner la dominance des hommes ou domination masculine (p. 9). Les auteurs citent les travaux de Margery Wolf (1972) et de Deniz Kandiyoti (1988), car ils s’intéressent à la façon dont les femmes sont à la fois des instruments du pouvoir masculin et des agents qui manœuvrent dans un système de pouvoir biaisé en leur défaveur.

Pour résumer, l'argument du livre repose sur la distinction entre, d'un côté, le sens étroit et classique du terme "patriarcat» qui englobe génération et genre, et, de l'autre, le sens plus large et moderne qui désigne directement la domination masculine et est fondé spécifiquement sur le genre. Gonçalo Santos et Stevan Harrell indiquent qu'ils reprennent dans cet ouvrage la notion de "patriarcat chinois classique» en termes néo-wébériens, à savoir : «un système hiérarchique de relations domestiques qui inclut de multiples intersections de structures inégalitaires telles que les inégalités de genre et de génération, parmi d'autres» (cité p. 10).

Â l'intérieur du patriarcat chinois, les femmes peuvent éventuellement être des «patriarches féminins» (p. 11), mais leur pouvoir est presque toujours restreint à la sphère domestique, tandis que la hiérarchie de genre s'impose dans la sphère publique et politique où les hommes prédominent, tout comme dans la littérature, les arts et le commerce (id.). Ce système d'autorité traditionnelle «que nous appelons le patriarcat classique» (p. 12), tirant sa force de fondements économiques, institutionnels et idéologiques extérieurs à la sphère domestique, est soutenu par deux institutions importantes: la parenté patrilinéaire étendue et les monopoles masculins dans les positions de pouvoir public. L'héritage masculin et la résidence virilocale donnent le contrôle de la propriété aux hommes et l'opportunité de la solidarité aux parents agnatiques (id.). Les jeunes et les femmes ont pourtant toujours une certaine marge de manœuvre au sein de ce système, notamment quand les femmes deviennent des belles-mères, car elles profitent alors de leur âge pour recevoir des marques de respect filial à côté de l'idéologie de la subordination des femmes (p. 13). Toujours selon les auteurs, il s'agit là des "points faibles» de «l'orthodoxie patriarcale stricte» permettant aux jeunes et aux femmes d'obtenir eux aussi un peu de pouvoir (p. 14).

\section{Catherine Capdeville-Zeng}


Avec la révolution communiste de 1949, le parti proclame l'égalité de genre et de génération, cherchant ainsi à réaliser la prophétie de Engels selon laquelle l'abolition de la propriété et le travail des femmes mettraient fin au pouvoir des hommes dans le mariage et à celui des anciens sur les plus jeunes au sein de la famille, tout en faisant disparaître la tyrannie du patrilignage dans les familles rurales ordinaires (p. 15). Cependant, en dépit de cette révolution de la famille, beaucoup de normes patriarcales ont perduré. Par exemple, les parents gardent le dernier mot en matière de mariage de leurs enfants, le travail des femmes continue à être sous-évalué, le mariage virilocal persiste ainsi que le prix de la fiancée, qui augmente même. Le fait que la propriété ait été collectivisée a eu finalement assez peu d'influence, parce que l'autorité est restée concentrée dans les mains des patriarches et des cadres masculins.

La période de réformes qui succède à l'ère maoïste entraîne une transformation sociale profonde liée au fait que la Chine urbaine ne peut plus être incluse dans le schéma des structures patriarcales classiques des relations matrimoniales. L'axe de génération perd de l'importance aux niveaux politique et économique (mais prime toujours en matière d'émotions et d'obligations) ; l'axe du genre reste dominé par les hommes, bien qu'il ne s'appuie plus comme avant sur les piliers traditionnels de la virilocalité, de l'héritage patrilinéaire et du pouvoir institutionnel des patriarches (p. 19). Le système patriarcal classique est en déclin dans les campagnes, la famille étendue et le «lignage corporate» ont régressé, et c'est la famille nucléaire qui s'est développée.

Avec ces transformations, des configurations patriarcales nouvelles émergent, mais tout en étant "très différentes de ce que les théories standard de la modernisation pouvaient prédire» (p. 31), elles-mêmes inspirées par les discours et expériences occidentales, qui postulaient « la fin du patriarcat au sens classique de Weber et le développement de la famille nucléaire et de l'individualisme affectif» (p. 33). La forme moderne du patriarcat fondée seulement sur le genre ne convient pas à la Chine «où la modernité n'a pas été accompagnée d'un déclin de l'importance des liens multigénérationnels» (id.), et où les inégalités de genre restent dès lors étroitement reliées aux inégalités de génération. Le modèle de patriarcat développé dans ce livre envisage donc "la modernité non comme la fin du patriarcat mais comme sa transformation» (id.). La perspective revendiquée est celle des "approches intersectionnelles de la troisième vague féministe (third-wave feminist intersectional approaches)", car elle se situe dans l'analyse des inégalités au niveau micro de la sphère familiale dans le contexte d'interactions inégalitaires multiples (p. 34). 
Le système émergent du patriarcat chinois se caractérise par les traits suivants (pp. 31-32):

- la domination des hommes, ou androcentrisme (andrarchy), demeure persistante;

- l'idéologie moderne individualiste d'égalité de genre et de génération coexiste avec la configuration classique patriarcale;

- la nouvelle configuration est construite autour de l'impératif du mariage et de la procréation;

- l'État s'est retiré des formes antérieures de sécurité sociale, de sorte que la famille doit maintenant assumer plus d'obligations;

- l'axe de génération n'a pas disparu mais s'est affaibli;

- le mariage est désormais défini comme une relation contractuelle reposant sur une satisfaction émotionnelle individuelle, incluant aussi des transactions monétaires (appartement/maison);

- la loi stipule que le cadre légitime de la reproduction est le mariage libre, hétérosexuel et monogame;

- les sentiments et l'affection ont remplacé partiellement l'obligation à la base des relations entre générations et entre sexes.

En résumé, la thèse soutenue dans l'ouvrage est la suivante: le terme «patriarcat» continue de caractériser, à un niveau général, la société chinoise, bien que les structures d'intersections multiples aient changé, puisque même si les asymétries de générations ne sont plus soumises à des forces institutionnelles coercitives, elles n'en restent pas moins vitales pour la famille et l'ordre social; de la même manière, bien que l'axe de genre ait lui aussi été reconfiguré, il n'en reste pas moins dominé par les hommes. Or, ce système émergent, combinant des traits anciens et des traits nouveaux, se fonde toujours sur les questions de l'égalité et de l'inégalité, centrales pour la démonstration, qui amalgament patriarcat et inégalité et les opposent à égalité et individualisme, sans considérer que les distinctions de statut sont inhérentes aux sociétés, même à celles qui se déclarent les plus individualistes. Du fait donc que les inégalités demeurent, le patriarcat demeure lui aussi.

Par ailleurs, l'usage inconditionnel du terme "patriarcat» est particulièrement frappant, appliqué uniformément à tous les lieux et à toutes les époques, nonobstant les «transformations" qui n'altèrent pas ce système. Ce mot issu de la culture occidentale est ainsi étendu à la Chine sans qu'aucune analyse des termes chinois correspondants ne soit entreprise. Certes, la note 12 (p. 35) rapporte deux termes utilisés en chinois pour désigner le patriarcat, traduits à partir de langues occidentales au XIX $^{\mathrm{e}}$ siècle d'après l'usage du japonais ${ }^{3}$ :

3. Les termes occidentaux ont d'abord été traduits par les Japonais, avant d'être repris en chinois, la Chine ayant été plus tardive que le Japon pour les traductions des pensées occidentales. 
fuquan zhi 父权制 (“système de pouvoir du/des père[s]») et jiazhang zhi 家长制 («système de chef de famille»). Mais, la même note infrapaginale signale que l'emploi de ces termes aujourd'hui reste limité aux seuls milieux éduqués des classes moyennes urbaines, ainsi qu'à une certaine et mystérieuse Chinese scholarship. Or, on peut se demander s'il est légitime d'utiliser un terme occidental dans un contexte chinois sans le questionner au préalable. Le recours au seul English Oxford Dictionary pour obtenir les définitions de cette notion indique que ce dictionnaire anglais est considéré par les auteurs comme ayant une autorité universelle. Quant à la convocation de grands noms tels que Morgan, cité dans les définitions évoquées, ou encore Weber, elle semble suffire à infuser une caution à ce mot, qui peut ainsi être employé comme un étendard globalisant. Enfin, aucune personne issue de cette Chinese scholarship mentionnée dans la note 12 n'a été invitée à discuter pour s'approprier ce mot "patriarcat ${ }^{4}$, qui, à ma connaissance, n'est pas non plus un concept central de l'anthropologie chinoise contemporaine (il est, par exemple, absent des travaux de Wang Mingming ${ }^{5}$ ).

Voyons plus avant les apories de ce concept étendu à la société chinoise en nous tournant vers différentes sources anthropologiques et sinologiques qui peuvent entrer en résonance avec l'ouvrage.

\section{Le terme "patriarcat" et la société chinoise}

Rappelons, en premier lieu, que le mot "patriarcat» est dérivé du grec ancien patriarkhês signifiant "patriarche" au sens de "chef de famille». Il fut utilisé dans l'histoire juive pour désigner les personnages bibliques, avant de devenir dans l'Église chrétienne un titre synonyme d'évêque, le patriarche pouvant alors être nommé ou élu. Cette histoire vient ainsi contredire l'idée moderne selon laquelle le patriarcat serait la concrétisation d'une forme de parenté se transmettant dans la lignée patrilinéaire et incluant un pouvoir coercitif, tout en attestant la transformation du sens de ce mot au cours des siècles. Il n'y a donc pas «un" patriarcat qui serait universel à toutes les époques et à toutes les sociétés, mais bien "des» patriarcats particuliers. Cela a été noté par l'historien sinologue Léon Vandermeersch, qui étudia la Chine ancienne:

"[L'organisation ancienne chinoise] fait apparaître la royauté chinoise traditionnelle comme un patriarcat, mais au sens très particulier d'un "patriarcat institué non par superposition aux pouvoirs respectifs de tous les pères du pouvoir supérieur d'un patriarche, mais par éviction de tous les pères de leur position paternelle, réservée au seul roi”" (cité in Billeter 1991: 878).

4. Les trois auteurs de l'ouvrage portant un nom chinois sont diplômés d'universités anglo-saxonnes ou exercent hors de Chine.

5. Wang Mingming est l'anthropologue chinois actuellement le plus en vue, et le plus prolifique, qui a notamment publié en anglais Empire and Local Worlds (2009) et The West as the Other (2014). 
Le patriarcat romain et le patriarcat chinois ancien sont donc bien différents, les hommes étant, en Chine ancienne sous la dynastie Shang, dans la position de fils par rapport au père-roi.

En Occident, c'est dans la seconde moitié du XIx ${ }^{\mathrm{e}}$ siècle, que le mot "patriarcat» ainsi que son pendant qui apparaît alors, le «matriarcat», commencent à être employés pour désigner un certain type de familles, propres à un certain type de sociétés. Dans le contexte du courant appelé l'«évolutionnisme» qui imprègne tous les domaines de la pensée au $\mathrm{XIX}^{\mathrm{e}}$ siècle, et notamment l'anthropologie sociale naissante, des théories sont élaborées par des penseurs comme Charles Fourrier (1830), puis, et surtout, par l'anthropologue américain Lewis Henry Morgan dans Ancient Society (1877), et enfin, par le théoricien marxiste Friedrich Engels dans L'Origine de la famille, de la propriété privée et de l'État (1884). Dans leurs écrits, le terme "patriarcat» renvoie à "une forme d'organisation sociale et juridique fondée sur la détention de l'autorité par les hommes, à l'exclusion explicite des femmes» (cité in Bonte \& Izard 2002 [1991] : 455). Le terme «matriarcat» se développe en parallèle, désignant quant à lui une organisation sociale fondée sur le pouvoir des femmes. Selon l'anthropologue suisse Johan Jacob Bachofen, qui s'appuie sur l'étude de mythes grecs dans Le Droit maternel (1996 [1861]), un matriarcat originel aurait existé à l'aube des sociétés humaines. Morgan puis Engels reprennent cette idée selon laquelle les premières sociétés humaines auraient été matriarcales avant de devenir patriarcales, suivant en cela une loi évolutionniste faisant passer les sociétés par différents stades de développement accompagnés d'organisations familiales particulières. Or, les idées évolutionnistes ont été largement battues en brèche depuis et, aujourd'hui, nul ne se revendique de cette tendance en anthropologie. En dépit de leur apport fondateur pour la science anthropologique, certaines propositions théoriques de Morgan, comme celles développées autour du patriarcat, peuvent donc être discutées. De plus, l'emploi de ce terme en tant que concept-clé du marxisme en référence aux travaux d'Engels doit inciter à la plus grande prudence quant à son utilisation récurrente.

Max Weber est lui aussi abondamment cité dans l'ouvrage, car il a fait du patriarcat une notion importante dans sa conception des différentes formes de domination à l'œuvre dans les sociétés, défendant notamment l'idée qu'il incarne un pouvoir absolu, coercitif, autoritaire. C'est dans cette idée que les auteurs du livre recensé ici utilisent l'expression «patriarcat classique chinois " pour désigner la famille chinoise à des époques relativement modernes (bien que s'appuyant sur la longue tradition chinoise) : la fin de la dynastie des Qing (XIX ${ }^{\mathrm{e}}$ siècle), la période républicaine (1911-1949) et la période maoïste révolutionnaire (1949-1978). Il est sous-entendu que la

\section{Catherine Capdeville-Zeng}


société chinoise "classique» posséderait une formule familiale «totalitaire». L'accent est mis sur la notion de "pouvoir", non seulement des pères, mais aussi de tous les hommes, ainsi que de quelques femmes, les belles-mères (mères de fils), qui oppriment les autres femmes, et particulièrement leurs belles-filles.

La patrilinéarité du système de parenté chinois est reliée au patriarcat, mais aucun des contributeurs n'a enquêté sur les rapports de parenté dans leur globalité, bien qu'ils évoquent fréquemment les rapports personnels maris-femmes, ou belles-filles-belles-mères, etc. Morgan déjà avait lié la descendance patrilinéaire au patriarcat et cet amalgame est donc reproduit ici. Dans le même ordre d'idées, les auteurs se montrent aussi très influencés par les conceptions des confucianistes chinois, dont ils semblent partager la vision hiérarchique des relations entre les sexes. En effet, la Chine ancienne a pu s'honorer d'avoir "fait société» à partir du moment où l'ordre social patrilinéaire a été instauré, justifiant ainsi la mainmise du "patriarcat».

Plusieurs textes classiques chinois, dont le Zhuangzi, le Lüshi Chunqiu et le Liji, indiquent que, dans le passé, les hommes connaissaient leur mère mais pas leur père, et qu'ils vivaient donc dans un «grégarisme bestial et une promiscuité sexuelle» (Vandermeersch 1991: 60), une idée qui rejoint tout à fait celle des penseurs occidentaux évolutionnistes du XIX ${ }^{\mathrm{e}}$ siècle. Léon Vandermeersch ajoute que "c'est au moment où le fils reconnaît son père, autrement dit où le mariage est institué, que commence la civilisation, disaient les anciens auteurs» (Ibid.: 67). Le mariage était autrefois, en effet, le seul moyen disponible aux hommes pour avoir l'assurance, en l'absence de tests génétiques, qu'ils étaient bien le père de leur enfant. Reconnaître ainsi une ligne de descendance (par les hommes), c'est fonder l'ordre patrilinéaire, et c'est aussi octroyer aux hommes la prépondérance dans la filiation. De plus, la Chine a adjoint au système patrilinéaire celui de la «hiérarchie sociale» prônée dans le confucianisme, avec des rapports entre les sexes asymétriques, les femmes étant les subordonnées des hommes. C’est pourquoi un écrivain qui étudie le confucianisme comme Alfred Doeblin s'autorise, en 1947, à nommer la subordination des femmes chinoises un "patriarcat» ${ }^{6}$.

Il est parfaitement exact que la société chinoise est d'essence hiérarchique (comme beaucoup de sociétés d'ailleurs, jusqu'à l'avènement de l'idéologie moderne égalitaire). La hiérarchie est conceptualisée en Chine principalement dans les relations interpersonnelles. Les fameuses «cinq relations» (wulun 五伦) humaines fondamentales du confucianisme établies par le

6. «[La femme] doit se subordonner à l'homme. Nous sommes à l'époque d'un patriarcat rigide» (Doeblin 1947: 40). 
penseur Mencius - père-fils, prince-ministre, mari-femme, aîné-cadet, ami et compagnon - expriment ainsi la totalité des rapports humains sur le mode de la complémentarité des positions hiérarchisées à l'intérieur d'une paire (cf. Mencius 2003). Cette hiérarchie sociale, en termes chinois, n'est pas comprise comme fonctionnant seulement sur les axes de genre et de génération, car elle opère dans toutes les relations sociales. Ce qui est essentiel pour la pensée chinoise confucianiste, ce n'est pas tant la conception d'une supériorité paternelle, mais l'existence de distinctions de statut et l'idée que tout rapport humain est hiérarchisé. Le terme "patriarcat» au sens wébérien est donc fallacieux parce qu'il élargit le pouvoir du père aux autres relations sociales, devenant ainsi l'argument central de la domination des hommes sur les femmes. En Chine, ce pouvoir du père, certes fondamental, ne s'étend cependant pas à toutes les autres relations: sur un plan politique, devant le roi, les hommes sont effectivement en position de fils (cf. supra), néanmoins, sur le plan privé, devant leurs femmes, ils sont des maris. Or, si les différences de statut sont communes à toutes les paires hiérarchisées, selon Mencius, un père et son fils sont liés par la "proximité» (qin 亲), tandis qu'entre un mari et sa femme prédomine la «distinction» (bie 别) . Dire que les hommes devaient être en situation de "père» de leur femme reste donc à démontrer. Accoler une relation de filiation à une relation entre les sexes est un artifice facile, mais dont l'évidence n'est pas directe.

En outre, la relation hiérarchique induit la réciprocité des devoirs: le prince doit s'occuper de son royaume en échange de la loyauté de ses sujets, les parents doivent se consacrer à l'éducation de leurs enfants qui, de leur côté, doivent soutenir leurs parents devenus âgés, les aînés reçoivent le respect des cadets mais leur doivent assistance en retour, etc. En raison de cette complémentarité même, il est difficile d'accepter de considérer cette famille chinoise "patriarcale» comme le lieu d'un pouvoir oppressif et exclusif des pères. En vérité, voir le patriarcat comme un système d'oppression ou de "coercition» (p. 32), c'est imposer une vision occidentale égalitaire des relations sociales qui rend toutes les autres incompréhensibles, insurmontables, voire répréhensibles. Parmi elles, la relation mari-femme et, plus généralement, la relation homme-femme, est peu à peu devenue la plus problématique, peut-être parce qu'elle suppose la subordination des femmes, considérée, avec l'avènement du communisme en Chine, comme la conséquence du rapport de domination illimitée exercé par les hommes.

7. Dans sa traduction de Mencius, André Lévy donne au terme qin le sens de «affection » : "Les cinq relations humaines fondamentales: l'affection entre père et fils, l'équité entre le souverain et le sujet, la distinction entre hommes et femmes, la hiérarchie des âges entre jeunes et aînés, la confiance entre amis» (cf. Mencius 2003: 87). Mais, comme le terme qin signifie en premier lieu la «proximité» et qu'il a ce sens dans les relations de parenté, je le préfere à celui choisi par ce traducteur. 
Après la révolution de 1949 et la loi sur le mariage de 1950, des slogans tels que «Hommes et femmes sont égaux» et "Les femmes sont la moitié du ciel», ou bien des pratiques comme le développement de l'emploi salarié des femmes sont apparus avec pour objectif d'émanciper les femmes.

Quels que soient les résultats de ces réformes engagées dans les années 1950, il ressort que le lien ancien comme moderne entre patrilinéarité et patriarcat ne va pas automatiquement de soi, nonobstant ce qu'en disait Morgan. Même si, en Chine comme ailleurs, les hommes occupent les fonctions politiques, religieuses, économiques de premier plan, et que les femmes ont été reléguées dans la sphère domestique et dans les tâches secondaires, cela ne signifie pas que le pouvoir d'un sexe sur l'autre découle automatiquement du système de filiation.

Il peut être intéressant de rappeler, à propos des formes de la filiation en lien avec celles du mariage, les travaux du sinologue "préstructuraliste» Marcel Granet, qui a montré, pour la Chine ancienne, l'évolution du système matrimonial depuis le système du chassé-croisé (ensuite appelé «échange restreint " par Claude Lévi-Strauss) jusqu'au système de l'échange différé ("échange généralisé» selon Lévi-Strauss). Dans ses Catégories matrimoniales et relations de proximité dans la Chine ancienne publiées en 1939, Granet avait élaboré le schéma scientifique de ce système d'échange à partir de sa lecture des classiques chinois. Repris ensuite tels quels par Lévi-Strauss dans les Structures élémentaires de la parenté (1949), les faits chinois anciens ont ainsi joué un rôle déterminant dans la théorie structurale. Granet a clairement dépeint, dans ses Catégories, l'importance de deux formes de filiations opérant à l'intérieur du système d'échange généralisé qui organise les mariages entre cousins croisés matrilatéraux, où, à une filiation patrilinéaire mise en avant, s'adjoint une filiation matrilinéaire tout aussi importante sur le plan théorique pour la constitution de ce système, mais que les Chinois ont choisi de ne pas reconnaître explicitement en privilégiant la patrilinéarité, à partir de la fin des Zhou et du début de l'empire (221 av. J.-C.).

Sur la question du lien entre filiation, mariage et domination des femmes, le point de vue structuraliste développé par Lévi-Strauss, avec certaines formules-clés des Structures, telles que "ce sont les hommes qui échangent les femmes et non le contraire» (1949: 134) ou "la relation d'échange [...] s'établit entre deux groupes d'hommes et la femme y figure comme un des objets de l'échange» (Ibid.: 137), a été très couramment compris à tort comme une reconnaissance d'une discrimination des femmes par les hommes. Car Lévi-Strauss ajoute aussi ceci : 
«[...] c'est que l'autorité politique, ou simplement sociale, appartient toujours aux hommes, et cette priorité masculine présente un caractère constant, qu'elle s'accommode d'un mode de filiation bilinéaire ou matrilinéaire, dans la majorité des sociétés les plus primitives, ou qu'elle impose son modèle à tous les aspects de la vie sociale, comme c'est le cas dans des groupes plus développés» (Ibid.: 136).

Lévi-Strauss reconnait donc, de façon explicite, que la "priorité masculine» n'est pas directement inscrite dans le mode de filiation, puisque la forme particulière de ce dernier ne la modifie pas, mais qu'elle découle plutôt de «l'autorité politique ou sociale».

Selon François Héran, qui a consacré plusieurs chapitres de son livre Figures de la parenté (2009) à l'apport de Granet au structuralisme, celui-ci admettait parfaitement que le modèle du système de l'échange généralisé puisse être réversible, les femmes échangeant les hommes, car le schéma des relations entre groupes fonctionnerait également dans ce sens. Or, historiquement, ce sont les hommes qui ont échangé des femmes, et non l'inverse: "[Granet] décrit avec force la suprématie masculine. Celle-ci ne relève pas d'une relation fondamentale, comme le prétend Lévi-Strauss, mais d'une pratique historique» (Ibid.: 453). S'il s'agit donc d'une pratique circonstancielle, cela implique logiquement qu'elle pourrait être amenée à changer.

Marcel Granet a en outre une conception chinoise du statut des femmes, pour laquelle leur subordination n'est pas directement consécutive à un système "oppressif», puisqu'elles sont tout autant des sujets de la société que les hommes. On peut signaler à cet égard que les termes de parenté chinois pour désigner les types de mariages sont, non pas comme chez Lévi-Strauss «mariage matrilatéral», mais "mariage entre la fille de l'oncle maternel et le fils de la tante paternelle», et non pas "mariage patrilatéral», mais «mariage entre la fille de la tante paternelle et du fils de l'oncle paternel». Les expressions lévi-straussiennes «mariage patri- ou matrilatéral» sous-tendent une emprise masculine sur le mariage (Ego masculin se marie avec telle ou telle cousine) et s'opposent au point de vue chinois que l'on pourrait appeler «bilatéral», repris par Granet.

Pourtant, Granet avait lui aussi commencé par envisager que la société chinoise originelle était " matriarcale» ${ }^{8}$ (avant de devenir patrilinéaire), puis il a abandonné cette idée dans les Catégories, où il postule que le système du chassé-croisé (échange bilatéral) fut le premier. Quoi qu'il en ait été réellement, ce qui ressort clairement dans les textes classiques est l'apparition du système patrilinéaire chez les Han, qui relient celui-ci au mariage.

8. «[L]a Terre paraissait une Mère. Ainsi il y eut une époque où la terre habitée et appropriée n’eut que des attributs féminins. L'organisation était alors tout près d'être matriarcale. Puis, quand les laboureurs, créant les institutions agnatiques, devinrent les maîtres de cultures, les génies du sol apparurent doués de traits masculins» (1968 [1934]: 195). 
Je crois que l'essentiel du propos de Granet est plutôt sa mise en valeur de l'absolue complémentarité des sexes, et donc du couple, dans la pensée et les pratiques chinoises:

"Tout d'abord, cependant, le pouvoir fut détenu par un couple princier. Le Chef ne dit point qu'il est le père du peuple. Il prétend en être le "père et la mère". C'est avouer qu’il a concentré en lui l'autorité qui, jadis indivise, appartenait à un ménage» (1968 [1934]: 210).

Ici, l'autorité prévaut sur le pouvoir, un terme qui ne sert qu'à désigner le gouvernement et non la façon de gouverner, et ce n'est pas l'homme seul qui se distingue, mais le couple composé des deux sexes, malgré leur séparation dans la vie quotidienne. Le privilège masculin est donc de détenir une «autorité» plus grande et publique, mais il ne s'agit pas d'un "pouvoir coercitif» exercé par un sexe sur l'autre.

Si l'on se réfère maintenant à des travaux plus récents, on constate par exemple que le célèbre anthropologue et réformateur chinois Fei Xiaotong, notamment dans le chapitre "Entre hommes et femmes il y a seulement des différences» de son grand livre Xiangtu Zhongguo (乡土中国 [La Terre chinoise]) écrit en $1945^{\circ}$, n'indique aucune "oppression" des premiers sur les secondes. L'esprit apollonien qui règne dans la Chine rurale n'encourage pas l'expression des émotions sentimentales, qui sont réprouvées par le système lignager, privilégiant les compagnonnages entre individus de même sexe. Ce qui caractérise les relations entre hommes et femmes est donc moins la sujétion que la distance. La restriction des libertés est le fait des groupes dominants - surtout les lignages - envers leurs membres, quel que soit leur sexe, bien que les hommes soient en position de supériorité.

L'évolution de la place des femmes dans l'histoire chinoise est présentée dans l'excellent article de Nicolas Zufferey, «La condition traditionnelle féminine en Chine: état de la recherche» (2003). Cet auteur y explique que la perception de la femme chinoise comme étant l'exact opposé de la femme occidentale présumée "libre» est une véritable "invention" idéologique des réformateurs, devenus révolutionnaires au milieu du $\mathrm{xx}^{\mathrm{e}}$ siècle. Cette nouvelle interprétation a permis aux communistes chinois, se fondant sur les idées occidentales, de justifier leur révolution, qui aurait «libéré» les femmes du patriarcat.

Plus encore, le consensus sur le supposé asservissement des femmes chinoises est aussi battu en brèche par d'autres chercheurs anglo-saxons. Ainsi l'anthropologue anglais Charles Stafford intitule-t-il un de ses articles "Actually Existing Chinese Matriarchy» (2009), dans lequel il

9. Ce livre n'a jamais été traduit en français, seulement en anglais sous le titre From the Soil (1992), mais en manquant la portée des concepts-clés. 
décrit le "pouvoir» des femmes chinoises et taiwanaises dans les domaines économiques, religieux et domestiques, où elles sont les "pivots" des relations de parenté, que celles-ci soient agnatiques ou d'affinité. Il termine son article en évoquant l'étude de Gail Hershatter (2003), par laquelle elle montre que c'est l'activisme politique en Chine, au début du $\mathrm{XX}^{\mathrm{e}}$ siècle, qui est à l'origine du jugement négatif que suscitent l'espace traditionnel domestique et la place qu'y tiennent les femmes, aboutissant à "priver cet espace de son statut causal [dans la vie sociale]» (2009: 150). Charles Stafford se demande alors si l'idéologie socialiste chinoise «n'a pas influencé les perspectives anthropologiques, nous conduisant à interpréter de façon erronée le rôle des femmes tant maintenant que dans le passé» (Ibid.: 151).

Finissons cette rapide revue de la littérature spécialisée en citant l'excellent livre de l'anthropologue chinoise Li Xia, Natal Family and Married-in Family. Women's Living Space and Backstage Power in a North China Village (2010), qui dépeint le "pouvoir» effectif des femmes dans un village chinois.

Il apparaît donc que la question du "pouvoir» des hommes sur les femmes en Chine est éminemment complexe et donne lieu à des interprétations différentes. Instiller une vision de la domination à tous les rapports humains est une extension de la pensée néo-wébérienne, revendiquée par l'ouvrage (p. 10). Certes, l'État communiste chinois actuel présente tous les aspects d'un régime de domination, que l'on peut considérer comme totalitaire, mais cela ne signifie pas pour autant que les rapports familiaux en Chine reproduisaient déjà ce modèle dans le passé, ni qu'ils s'en inspirent encore aujourd'hui. Cette question va maintenant être étudiée à travers ce qu'en disent les contributeurs de l'ouvrage, qui ont observé les rapports entre hommes et femmes dans la Chine contemporaine.

\section{Les enquêtes ethnographiques de l'ouvrage}

Les contributions rassemblées dans cet ouvrage sont réparties en trois grandes parties, correspondant chacune à un contexte d'émergence de la nouvelle forme de patriarcat en Chine: la campagne, la ville et les nouvelles technologies. Elles ont presque toutes été rédigées à partir de données collectées lors d'enquêtes de terrain longues et intensives, certains auteurs y revenant année après année. Cependant, les expériences personnelles ou la façon dont les enquêtes ont été menées ne sont que peu détaillées, et aucune réflexion n'est proposée sur les questions de méthode. Certains auteurs ne se basent que sur des enquêtes statistiques ou des articles de la presse chinoise. Plusieurs textes sont complétés par des extraits d'entretiens et de courtes biographies venant à l'appui de la démonstration.

\section{Catherine Capdeville-Zeng}


Ce sont donc les résultats de ces enquêtes ethnographiques que nous examinerons dans cette partie, qu'ils soient porteurs d'informations intéressantes sur la société chinoise ou le fruit de généralisations hâtives qui, nous le verrons, m'inciteront à émettre quelques réserves.

\section{La reconfiguration du patriarcat à la campagne}

Le premier texte de Melissa J. Brown, intitulé «L'aide obéissante: masquer les contributions économiques des femmes rurales» (chap. I, pp. 39-58), décrit la façon dont le travail des femmes est toujours sous-évalué en donnant l'impression qu'elles ne travaillent pas vraiment autant que les hommes, qu'elles ne font que les «aider» (bangmang 帮忙), expression qui sert à «rationaliser le patriarcat» (p. 40) et "fait partie intégrante de la parenté chinoise» (p. 41). Ayant mené une enquête extensive à partir d'entretiens dans onze provinces, l'auteure note que ce ne sont pas seulement les familles et les hommes qui utilisent cette expression, mais bien les femmes aussi, qui se sous-évaluent donc elles-mêmes. Il en est ainsi parce que leur «sens du devoir» (p. 55) les pousse à donner leurs revenus à leur famille natale avant leur mariage, puis à les verser dans l'escarcelle du foyer après. De sorte que «les femmes chinoises se montrent sensibles aux idéologies patriarcales anciennes» (p. 55), voire elles les soutiennent.

Dans «De donneurs de soins à fardeaux financiers: le changement du rôle des fils et du choix de reproduction en Chine rurale du Nord-Est» (chap. II, pp. 59-73), Lihong Shi commence par indiquer que: «La tradition patrilinéaire et patriarcale chez les Chinois a rendu les fils économiquement responsables pour subvenir aux besoins de leurs parents dans leur vieillesse et culturellement cruciaux pour perpétuer la lignée ancestrale» (p. 59). C'est pourquoi les parents chinois ont marqué une préférence forte pour les garçons, qui leur sont indispensables. Or, depuis les années 1990, dans le village étudié de la province du Liaoning, un nombre significatif de couples ayant donné naissance à une seule fille a décidé de ne pas profiter des assouplissements de la politique de l'enfant unique qui leur permettaient de retenter leur chance avec un deuxième enfant. Cela s'explique par l'affaiblissement de l'idéologie patrilinéaire, qui rend moins stigmatisant, de nos jours, le fait de ne pas avoir de fils. Plus encore, les parents n'ont plus confiance en leurs fils pour qu'ils les prennent en charge durant leur vieillesse, ces derniers devenant même des fardeaux financiers au fur et à mesure qu'augmente le coût de leurs mariages. D’un "désir de fils», le village étudié est donc passé à la "peur des fils» (p. 63). En outre, les filles font souvent preuve de davantage de dévouement filial que les belles-filles. Cependant, cette évolution n’entraîne pas la fin du patriarcat. L'auteure l'explicite par le fait que, quand une fiancée demande un prix élevé à ses 
beaux-parents pour son mariage, sous-entendant qu'il s'agit d'un paiement II4 pour ses futurs enfants et son travail, la logique sous-jacente procède toujours de la tradition chinoise patrilinéaire. Cette situation crée un paradoxe: parce que les femmes rurales utilisent l'idéologie patriarcale et patrilinéaire pour atteindre leurs objectifs, parmi lesquels obtenir de l'argent pour soutenir leurs propres parents, elles contribuent par là même à renforcer l'idéal patrilinéaire et leur propre marchandisation dans le mariage.

Les résultats de l'enquête menée par Helena Obendiek dans la province du Gansu (chap. III : "Études supérieure, genre et soutien des aînés en Chine rurale du Nord-Ouest», pp. 74-90) montrent que, contrairement à la précédente sur le Liaoning où les relations de pouvoir entre les genres et les générations ont été altérées, il y existe encore un investissement préférentiel des parents pour les garçons, et ce, toujours dans le souci de bénéficier de leur soutien dans leur vieil âge. Il apparaît aussi que les fils partis suivre un cursus universitaire en ville rencontrent davantage de difficultés que les filles, compte tenu de leur obligation de subvenir aux besoins des parents et des coûts des logements en ville. De leur côté, les filles bénéficient d'une plus grande indépendance, parce qu'elles n'ont pas la pression de devoir «rembourser» leurs parents pour leur investissement dans leurs études. Cela dit, comme elles restent liées aux obligations de leur mari, l'investissement des parents continue de favoriser finalement les fils: «Les femmes diplômées souffrent toujours des notions locales de la patrilinéarité qui limitent leur indépendance et une équité [de traitement] entre elles et leurs maris et petits amis» (p. 89).

Dans «Maternage multiple et migration en Chine rurale du Sud» (chap. IV, pp. 91-110), Gonçalo Santos décrit le phénomène massif des 61 millions d' "enfants laissés derrière" à la campagne (p. 93), chez leurs grands-parents, qui les gardent pour aider leurs propres enfants migrants dans les grandes villes. Ce phénomène de parenté de substitution n’est pas envisagé ici en tant que pathologie sociale, mais comme une fenêtre d'observation sur le rôle protecteur des grands-parents envers leurs petits-enfants, un comportement qui a une grande signification dans la vie familiale chinoise. Néanmoins, le maternage multiple n'est pas seulement une structure informelle d'aide familiale, "c'est aussi le lieu de l'exploitation patriarcale" (p. 94). En effet, bien que ce phénomène semble valoriser le pouvoir des femmes, dans les faits, en renforçant l'autorité de la génération supérieure et la définition d'un espace domestique réservé aux femmes, il contribue à accentuer les inégalités de genre et de génération (id.). En outre, ce sont prioritairement les grands-mères paternelles qui jouent le rôle de «mères multiples", et non les grands-mères maternelles. Ce modèle montre donc que la famille moderne nucléaire ou conjugale (dyade parents/enfants) ne 
s'est pas forcément imposée en Chine rurale, et que l'émancipation des jeunes ne doit pas se comprendre comme «le triomphe de la conjugalité» (notamment selon Yan Yunxiang 1997), mais « comme une reconfiguration des relations d'interdépendances de genre et de génération typiques des structures familiales patriarcales» (p. 106). La génération du milieu a gagné en autonomie, mais cela n'a pas entraîné l'abolition du contrat générationnel. De sorte que, si ces migrations des jeunes, y compris des jeunes femmes, pour travailler, ont bien transformé le système familial traditionnel, le système patriarcal ne disparaît pas pour autant mais est "restructuré» (p. 95). Cependant, dans le même temps, alors qu'autrefois les femmes pouvaient espérer obtenir du pouvoir en devenant des belles-mères, aujourd'hui, les belles-filles ne reconnaissent plus leur autorité, même lorsqu'elles ont la charge de leurs enfants. Alors, les grands-mères/belles-mères «se sentent flouées» (p. 107). Dans cette reconfiguration, les interdépendances entre générations sont certes un peu plus équilibrées, mais pour les filles chinoises locales, la vie hors du mariage reste toujours une «non-option» (p. 109).

Tous ces chapitres sur le monde rural montrent ainsi la réalité d'une «reconfiguration de l'ordre patriarcal», mettant en évidence une société en transition où les pratiques évoluent globalement, mais sans aboutir immédiatement à une indépendance et à une libéralisation par rapport aux rôles traditionnels. Cela se produit plus ou moins vite et plus ou moins nettement selon les personnes et les contextes, et, s'il est indéniable que les liens traditionnels tendent vers plus d'individualisation, il apparaît aussi que la famille représente toujours une institution où l'entraide reste fondamentale.

\section{Classe, genre et patriarcat dans la société urbaine}

Andrew Kipnis, dans sa très intéressante contribution intitulée "L'urbanisation et la transformation des pratiques de parenté dans le Shandong" (chap. V, pp. 113-128), analyse les évolutions des relations de parenté chez les urbains et «nouveaux urbains». Il indique que «la néolocalité, la parenté conçue comme bilatérale et une relation plus équilibrée entre les genres» n'ont pas remplacé automatiquement "la patrilinéarité, la virilocalité et le patriarcat» (p. 115); une transformation complexe de chacun de ces domaines a bien lieu, mais elle ne touche pas tout le monde de la même façon ni au même moment (p. 116). Sont passés en revue la résidence, l'héritage, les soins donnés aux enfants et aux parents âgés. Chez les migrants des villages proches de la ville de Zouping, la "viricentralité» reste de mise, les parents de l'homme jouant un rôle plus important que ceux de la femme; à l'inverse, chez les migrants venant de régions plus éloignées, les familles nucléaires pratiquent une résidence néolocale et les 
relations de parenté sont très simplifiées, la "viricentralité» et l'idéologie patrilinéaire déclinant fortement; pour les «villageois dans la ville» (qui a englobé en se développant les villages avoisinants), ceux qui ont le plus profité économiquement de ce rapprochement, le modèle "viricentrique» et patrilinéaire domine, bien qu'une place non négligeable soit accordée aux relations avec les affins. Le terme «viricentralité» est introduit pour désigner les pratiques de parenté toujours patrilinéaires de ceux qui quittent leur village natal, où ils travaillaient et résidaient au même endroit, pour aller vivre dans un espace industrialisé et urbain, où le travail et la résidence sont distincts (p. 126). Ces personnes gardent cependant des liens étroits avec leur village natal et continuent à avoir des relations et une idéologie patriarcales traditionnelles. Ceux qui s'en distancient le plus sont les migrants de régions éloignées. Ainsi la vie en ville est-elle très différente selon que l'on appartient à l'un ou l'autre de ces trois groupes d'habitants.

Dans «Être la femme parfaite de "monsieur Parfait" » (chap. VI, pp. 129145), Roberta Zavoretti suit le parcours d'une jeune infirmière de la ville de Nankin qui, pour se marier, abandonne son projet d'émigrer pour aller étudier la médecine et se coule dans une vie de famille, où elle se consacre totalement à son mari, puis à son enfant, avec l'aide des parents des deux côtés. Selon l'auteure, elle finit par reproduire les schémas patriarcaux spécifiques, à travers des pratiques sociales en mutation.

Dans «L'amour conjugal émergent, l'affection mutuelle et le pouvoir marital des femmes» (chap. VII, pp. 146-162), William Jankowiak et Xuan Li ont pu observer, à partir d'enquêtes menées respectivement dans les villes de Hohhot (Mongolie intérieure) et de Nanjing (Jiangsu), que:

«[...] quand le lien d'amour devient un idéal culturel dominant et une pratique préférentielle, les valeurs patriarcales qui soulignent la supériorité des hommes et femmes âgés sur leurs enfants et, dans un contexte plus localisé, la prééminence d'un mari sur sa femme ne peuvent plus se développer, et encore moins prospérer» (p. 146).

La jeune génération urbaine née sous l'ère de l'enfant unique a ainsi adopté le modèle du mariage "d'amour authentique», fondé sur des sentiments d'équité, une estime partagée et une appréciation mutuelle plus grande. C'est un "mariage émotionnellement égalitaire» construit autour d'un «langage de l'amour» par opposition à un «langage du devoir» (p. 147). Cet idéal de la relation amoureuse se manifeste dans les nouvelles pratiques de cour et dans le fait que les hommes et les femmes ne sont plus tenus de «jouer un rôle» (id.). Aujourd'hui, outre que les jeunes se marient donc beaucoup plus par amour qu'auparavant, l'intimité et l'affection peuvent davantage s'exprimer publiquement, et les femmes former des souhaits autrefois déniés par la société patriarcale. La pratique de la cour transforme 
profondément la structure intergénérationnelle d'autorité (p. 151). Les attentes et les responsabilités associées au fait de vivre dans une famille étendue ont laissé place à une relation dyadique où hommes et femmes, désormais perçus comme des agents individuels, construisent des liens de coopération mutuels où priment le respect et l'admiration, ou l'amour profond (p. 156). Les femmes ont accru leur pouvoir d'influence au sein du couple et jouent beaucoup moins le rôle de l'«épouse obéissante» (dutiful spouse) tout en attendant plus d'engagement affectif et émotionnel de la part de leur mari. Elles se sont affirmées comme des "managers» au sein de leur famille (p. 160) : «les nouvelles sources de pouvoir des femmes - le désir de leurs maris de les aimer - leur a permis de négocier les obligations auxquelles elles étaient autrefois astreintes envers leur belle-mère" (p. 161). Et le chapitre se conclut sur le constat que: «Les individus sont maintenant plus ou moins seuls dans leurs efforts pour trouver des satisfactions dans une culture qui n'est plus sûre d'elle-même» (p. 162).

L'étude suivante de Elisabeth L. Engebretsen, «Sous la pression: mariages contractuels entre gays et lesbiennes et leurs négociations patriarcales" (chap. VIII, pp. 163-181), évoque les faux mariages hétérosexuels, principalement observés à Pékin, arrangés entre homosexuels pour "répondre à la pression sociale intense sur le mariage» (p. 163). Cette pratique a donc l'apparence d'un vrai mariage, mais sans autre implication que celle de la reconnaissance publique de son existence. Ce genre de mariage contractuel est en conformité avec l'ordre social et familial, puisqu'il organise une stricte compartimentation entre une vie homosexuelle privée et une façade sociale hétérosexuelle (p. 164). Beaucoup d'homosexuels chinois, hommes et femmes, considèrent que ce mariage est "un compromis idéal entre désir personnel et devoir social et familial» (id.). L'auteure essaie de comprendre ce qui peut justifier la nécessité d'un tel compromis à une époque où l'individualisme et les valeurs modernes d'égalité et d'autonomie se répandent, en montrant qu'il s'explique par la pérennité des pressions sociales liées aux structures patriarcales. Ce type d'arrangement réaffirme la dominance de la famille hétérosexuelle normative en tant qu'unité sociale et morale de base. L'homosexualité doit rester invisible, et les lesbiennes et les gays qui pratiquent ce mariage s'épuisent à devoir jouer constamment un rôle. Des expressions spécifiques sont apparues pour se conformer à l'ordre hétérosexuel: par exemple, la "deuxième fille» (les parents adoptent le conjoint féminin de leur fille), «donner des soins aux parents de la conjointe», ou le terme neutre d' "amie» (p. 178). Finalement, selon l'auteure, «le mariage contractuel démontre que les idéologies patriarcales restent une structure normalisante fondamentale qui confirme l'inégalité dans les pratiques de parenté...» (p. 179). 
Harriet Evans, dans «Investissements patriarcaux: recherche d'autorité et de soutien masculin dans un quartier pauvre de Beijing [Pékin]» (chap. IX, pp. 182-198), présente trois exemples de familles dans lesquelles les femmes jouent un rôle prépondérant et où elles déploient des stratégies par rapport aux «figures patriarcales de leurs vies, incluant maris, pères et belles-mères" (p. 182). Leur investissement dans ces familles n'est pas fondé seulement sur des considérations pragmatiques, mais aussi sur une prise en compte de responsabilités et de sensibilités éthiques - être une personne morale, avec un sentiment de valeur personnelle marqué par le genre (id.). Les trois exemples diffèrent mais « convergent par leur soumission à l'autorité du chef de famille masculin du foyer, directement ou de façon oblique, et en sa présence ou son absence» (p. 195). «Les femmes ont de forts caractères, sont conscientes de leurs capacités et protègent leurs activités indépendantes, mais, en même temps, elles s'accommodent des pratiques et valeurs patriarcales pour des raisons pragmatiques, idéologiques et éthiques» (id.). "L'homme - dans ces cas, le mari, le fils et l'amoureux - devient le pivot central autour duquel s'articulent les attentes de la femme pour sa propre sécurité émotionnelle et matérielle» (id.). Ainsi, le "patriarcat apparaît comme récurrent [...], se manifestant dans les pratiques, croyances et attitudes des hommes et des femmes» (p. 197).

Comme dans le monde rural, la ville est le théâtre d'une évolution certaine des pratiques patriarcales, bien que les hiérarchies demeurent importantes, notamment dans les relations entre les deux sexes. Et, en ville aussi, l'entraide familiale et les rapports à l'intérieur des familles restent déterminants, de même que les individus ne sont pas encore parvenus à échapper à l'accomplissement de leur rôle social, comme le montre l'exemple du faux mariage des homosexuels.

\section{Nouvelles technologies, nouvelles institutions}

Cette troisième partie commence avec une contribution de Suzanne Gottschang, intitulée "Sortir le patriarcat du rétablissement postnatal» (chap. X, pp. 201-218), où elle étudie la transformation d'une tradition ancestrale chinoise appelée littéralement "S'asseoir un mois» (zuo yuezi 坐月子) et correspondant à la période de convalescence pendant laquelle une mère et son nouveau-né se remettent de l'accouchement et de ses suites sous le contrôle étroit de la belle-mère. Selon cette tradition, les nouvelles mères doivent régénérer leur corps, ce qui signifie observer certains interdits (ne pas se laver, ni le corps ni les cheveux, ne pas quitter la maison, ne pas avoir de relation sexuelle) et se soumettre à un régime strict (ne pas manger de nourritures froides ou crues, mais seulement certains aliments dûment sélectionnés, souvent à base de poulet). Si cette pratique avait

\section{Catherine Capdeville-Zeng}


pour objectif de protéger la mère et l'enfant, elle avait aussi pour finalité de "préserver les intérêts de la famille patrilinéaire et de tout l'entourage des dangers [que pouvaient constituer] les souillures féminines" après un accouchement (p. 205). Aujourd'hui, à l'époque des hôpitaux, des infirmières et des médecins, des "Centres zuo yuezi» ont vu le jour, servant à la fois de maisons de repos et d'établissements biomédicalisés. Ces centres n'éloignent pas forcément la belle-famille et ces jeunes mères doivent en plus relever les défis que le modèle de la famille nucléaire impose à la vie de couple, comme celui de retrouver au plus vite leur corps d'avant la maternité et s'efforcer de plaire à leur mari. Quant aux soins qui y sont prodigués aux nouveau-nés, ils ont aussi pour objectif de les préparer à être «de condition supérieure " car, contre l'idéologie soi-disant "patriarcale», les femmes ont plus que jamais le pouvoir de formater leurs enfants et d'influencer le cours de leur vie. Ces centres illustrent ainsi l'amalgame de l'ancien avec le nouveau, comme celui des principes de la médecine chinoise traditionnelle avec le compromis trouvé entre les générations pour que la mère et la bellemère redéfinissent ensemble leur place, avec pour résultat de «renforcer et reconfigurer les structures d'autorité et de pouvoir régissant les relations entre femmes jeunes et moins jeunes dans la famille» (p. 216).

Kerstin Klein, dans «Technologies de procréation assistée, don de sperme et parenté biologique» (chap. XI, pp. 219-233), présente ensuite les nouvelles conceptions des Chinois concernant la parenté dans un contexte de faible natalité, notamment la patrilinéarité biologique et sociale. Ne pas avoir d'enfant a longtemps été considéré comme un manquement au devoir filial dû aux ancêtres, c'est pourquoi l'histoire chinoise est emplie d'exemples de solutions alternatives pour devenir parents: adoption de fils d'agnats ou d'étrangers, mariage uxorilocal, adoption de filles pour les marier uxoricalement, concubines (p. 221). Face au nombre de moins en moins élevé d'enfants à adopter aujourd'hui, la PMA s'est développée, mais elle s'est ellemême heurtée au fait que le don de sperme est très controversé en Chine. Le manque de donneurs est généralement imputé à l'influence du système patrilinéaire et à la peur des hommes d'avoir des enfants d'inconnus. Bien que des banques de sperme aient maintenant une place dans de nombreuses universités (car les étudiants sont les donneurs les plus nombreux), le procédé est toujours perçu comme un acte honteux, peut-être en raison du tabou associé à la masturbation ou de l'incitation mystique traditionnelle faite aux hommes de contrôler leur essence vitale. Il apparaît donc que l'accent est désormais mis sur l'aspect biologique de la parenté, alors que l'adoption a toujours été pratiquée en Chine sans problème: «Il n’y a jamais eu en Chine la célébration, la promotion ou l'accord envers une continuité biologique en l'absence de continuité sociale» (p. 227). 
Le dernier texte de Hong Zhang, "Recalibrer la piété filiale: réaligner les intérêts de l'État, du marché et de la famille en Chine» (chap. XII, pp. 234-250), traite du soutien que les enfants se doivent d'apporter à leurs parents âgés. Fondée sur les principes patriarcaux, la piété filiale est restée une valeur culturelle centrale en Chine, malgré les transformations sociales. Cependant, une nouvelle industrie de "soins aux plus âgés » est apparue, entraînant un mouvement de marchandisation de cette vertu. L'État chinois a aussi cherché à réformer le système de sécurité sociale, comprenant des pensions de retraite et une allocation vieillesse de base pour les personnes sans ressources, tout en incitant au renforcement des valeurs filiales. Cette transformation intervient au moment où les filles, qui autrefois jouaient un rôle secondaire dans le soutien à leurs propres parents, les assistent maintenant de plus en plus. Ce phénomène s'observe en ville, mais Hong Zhang indique aussi que, dans un village de la province du Hubei, cette évolution s'inscrit dans une nouvelle forme de «mariage bilatéral» (liangbian dianli 两边典礼) ${ }^{10}$ (p. 241) impliquant une même obligation des filles de s'occuper de leurs parents. Il en résulte que la «tradition patriarcale valorisant l'importance des fils pour le soutien des parents âgés a été révisée dans la Chine contemporaine» (id.). Parallèlement, de plus en plus de parents cherchent des moyens de se prendre en charge de manière plus autonome pour ne pas être des fardeaux pour leurs enfants. On assiste donc à une plus grande indépendance de la génération âgée et à une plus grande réciprocité entre cette dernière et celle des enfants (p. 243). Avec "cette nouvelle piété filiale», la relation parents-enfants devient moins hiérarchique et plus équilibrée, et montre une perception un peu plus égalitaire des fils et des filles (p. 249).

Les nouvelles technologies et institutions permettent donc de remodeler les pratiques familiales anciennes, d'un côté en assouplissant les relations entre belles-mères et belles-filles autour de la naissance et en inventant une forme «bilatérale» de mariage, de l'autre, en réhabilitant la part biologique de la parenté alors que l'adoption était autrefois largement pratiquée, bien que dévalorisée dans le discours extrémiste confucianiste. De ces deux tendances découle le constat d'un renforcement des relations personnelles au détriment des relations normées, et d'une valorisation de l'individu.

10. Cette expression désigne la participation des deux familles à la cérémonie de mariage, n’engageant pas directement une forme nouvelle de parenté. On aurait aimé plus de détails sur ce que signifie cette expression pour les locaux qui l'emploient. 


\section{Commentaires sur les enquêtes}

J'organiserai mes commentaires autour de thèmes transversaux abordés

dans les différentes contributions que nous venons d'évoquer, en faisant appel à ma propre expérience anthropologique de la Chine et à des sources extérieures.

\section{La pérennité de la valeur famille, en ville et à la campagne}

Tous les chapitres de l'ouvrage montrent que les interdépendances familiales restent importantes: les enfants dépendent de leurs parents, ceux-ci de leurs fils, et parfois aussi de leurs filles, les couples semblent soudés. Cette interdépendance y est vue comme le signe du patriarcat.

Par exemple, la répartition des tâches entre grand-mères rurales, au profit des grand-mères paternelles, analysée par Gonçalo Santos (chap. IV), a bien pour effet de décharger les grands-parents d'une partie de ces soins et de les distribuer de part et d'autre en fonction du sexe de leurs enfants, et donc de structurer les rapports selon le sexe; ce déséquilibre ayant pour finalité de préserver le patriarcat. On peut augurer que si les grands-parents devaient s'occuper de tous leurs petits-enfants, qu'ils soient issus de leurs fils ou de leurs filles, l'entraide reposerait alors sur les relations individuelles entre les personnes de la famille, et non plus sur la norme de la distinction de sexe. Le présupposé de l'auteur est que de telles relations individuelles non fondées sur la distinction de sexe seraient plus équilibrées et signifieraient la fin du patriarcat.

Si cette distribution entre les sexes reste l'apanage de la campagne, elle est alors en contraste avec les observations de Roberta Zavoretti (chap. VI), qui dépeint la façon dont les deux familles parentales pèsent sur la vie d'un jeune couple urbain, alors que ce dernier réside chez les parents du garçon et que la jeune femme compte énormément sur sa propre famille maternelle pour la soutenir. Par différence avec le monde rural, ce sont donc bien ici les deux côtés parentaux qui interagissent avec le couple. Se démarquer de la notion de patriarcat aurait permis de mettre en évidence les implications urbaines de la notion de «famille» et d'éviter l'amalgame entre famille et patrilinéarité pour mieux prendre en compte les aspects bilatéraux. L'expression «négociations patriarcales» traduit les interdépendances mises en ouvre par les membres de la famille car, si la jeune femme n'est pas une victime car elle choisit et négocie la place qu'elle occupe, néanmoins, elle n'est pas du tout autonome.

La relation entre belle-mère et belle-fille au moment où cette dernière devient mère (chap. X) apparaît comme étant plus distanciée qu'auparavant, ce qui marque une adaptation appréciable au monde moderne des 
deux générations concernées, notamment lors du séjour dans les centres zuo yuezi. Pourtant, en envisageant ces établissements comme un «soutien au patriarcat", l'auteure semble sous-entendre que seul l'isolement de la nouvelle mère et de son bébé, sans aucune assistance de sa belle-mère, les libérerait de l'emprise patriarcale. On pourrait proposer, au contraire, de comprendre cette institution comme un lieu dédié à la relation sociale, en tant que relation humaine venant en soutien lors d'une naissance, et comme un moyen d'évitement de la solitude de la jeune mère démunie face à son bébé.

À la ville comme à la campagne, les enfants dépendent encore aujourd'hui de leurs parents pour financer leurs études, organiser leurs mariages, ou garder leurs propres enfants et, en retour, ils doivent toujours s'occuper de leurs aînés devenus âgés, même si une nouvelle offre d'établissements dédiés, fort onéreux, se met en place pour proposer une alternative au principe de piété filiale. L'entraide et l'interdépendance demeurent des valeurs fondamentales, mais tendent vers une distanciation des rôles traditionnels. Ainsi la famille reste-t-elle un ensemble englobant qui soutient ses membres, notamment dans le contexte de développement brutal du capitalisme économique chinois, où ils ne profitent plus du cadre des anciennes unités de travail et se retrouvent souvent seuls face au monde nouveau. La famille est également devenue le lieu d'une relation hiérarchisée, où les anciens conservent leur position respectée, en vertu de quoi leurs enfants leur doivent aussi une assistance. Les différences de génération, conçues dans le monde chinois comme étant d'essence naturelle et définies par l'ordre de la naissance, comptent toujours même si elles ont certainement été atténuées, car les parents chinois ont aujourd'hui souvent à cœur non seulement d'aider leurs enfants mais aussi de mettre en place avec eux une relation plus proche et chaleureuse. Tant les chapitres de ce livre sur les relations familiales chinoises que mon expérience personnelle montrent que la distinction entre parents et enfants ne suffit pas pour caractériser cette forme familiale comme une domination de génération inhérente à la notion de "patriarcat». En effet, ce ne sont pas seulement les pères et les hommes qui gouvernent ces familles, et les avis des mères ne doivent pas être minimisés. De sorte que la question est plutôt de savoir si l'entraide procède des seuls hommes représentatifs du patriarcat ou si elle n'est pas tout simplement constitutive de l'organisation sociale et familiale chinoise.

\section{La dévalorisation des femmes, pratiques et discours}

Le thème de la dévalorisation des filles fondée sur la distinction de sexe est relié au patriarcat que soutiendraient la plupart des acteurs chinois convoqués dans l'ouvrage. On se demande cependant si ce mot "patriarcat» et la situation qu'il désigne - des relations familiales inégalitaires - revêtent

\section{Catherine Capdeville-Zeng}


le même sens pour les enquêtés et pour les enquêteurs, étant donné que beaucoup d'informateurs, y compris des femmes, jugent les relations familiales comme globalement satisfaisantes.

Dans la contribution de Lihong Shi (chap. II), le "prix de la fiancée» (caili 彩礼, appelé parfois ailleurs une «dot indirecte») est présenté comme sous-tendant directement le pouvoir de la famille du garçon. Différentes études (par exemple, Cohen 2005) ont pourtant montré que cet échange, dans sa partie monétaire, revient prioritairement au nouveau couple sous la forme de dot, dont l'épouse est la gestionnaire, et non aux parents de cette dernière (qui peuvent certes en conserver une partie). L'objet de cette institution commune à de nombreuses sociétés, en plus de rembourser un tant soit peu la dette symbolique incommensurable contractée pour la venue d'une belle-fille, est aussi en Chine d'aider les jeunes mariés en leur donnant des fonds propres, sachant que l'époux et l'épouse ont des statuts différenciés à l'intérieur du couple, lui-même inscrit dans la lignée patrilinéaire. L'auteur semble ignorer que les filles chinoises doivent toujours, après leur mariage, continuer à offrir des dons rituels à leurs propres parents et à les aider en cas de besoin, même si cette aide était secondaire par rapport à celle demandée aux fils. Effectivement variable selon les régions, l'apport des filles est régulièrement minimisé ou tenu à l'écart par nombre de chercheurs, qui valorisent sciemment le poids du masculin, se fondant sur une appréciation quantitative des inégalités entre les aides dues selon le sexe. Plus encore, l'affection sincère que pourraient éprouver les filles pour leur famille d'origine n'est pas considérée comme un paramètre dont il faudrait tenir compte. Ce constat d'une dévalorisation extrême des filles provient donc en grande partie du fait que le système n'est pas étudié dans toutes ses ramifications et implications, mais aussi de ce qu'on appréhende individuellement les deux membres du couple, que l'institution du caili a pour visée de construire comme une entité duelle et indivisible.

Le thème de l'inégalité entre les sexes revient dans le chapitre III, centré sur les questions économiques et financières: bien que les filles ne soient pas tenues de rembourser leurs frais d'études en prenant soin de leurs parents âgés, ces derniers investissent malgré tout aussi massivement qu'ils le peuvent dans leur éducation. La rationalité des acteurs est dès lors mise à l'épreuve, montrant là encore que le critère économique du retour sur investissement ne peut à lui seul expliquer les actions, et que celui de l'affection devrait être mieux pris en compte.

À côté des pratiques, les analyses des discours des «femmes qui aident» (chap. I), relevant une autodévaluation de leur travail face à une surévaluation des apports des hommes, omettent les habitudes relationnelles chinoises de manipulations des hiérarchies sociales. Il est en effet courant chez les 
Chinois - et pas seulement chez les femmes - de se rabaisser intentionnellement pour «donner de la face» (du prestige) à son interlocuteur (un maître, un patron, un vieil homme ou une vieille femme, un mari, un invité, etc.). Cet usage est si commun en Chine, qu'il devrait être évoqué pour comprendre cette parole rituelle des femmes, sans compter qu'elles savent très bien que leur travail est essentiel sur le plan pratique. Parallèlement, la parole symétrique des hommes dévalorisant le travail des femmes pourrait être comprise comme une tentative d'afficher et de manifester leur statut, plutôt que comme la représentation d'une totale non-reconnaissance de la contribution des femmes. Si ces formulations de l'«aide» affirment la hiérarchie sociale entre les sexes, il importerait de mieux les analyser par rapport aux pratiques réelles et de ne pas s'y fier unilatéralement, parce qu'ils subordonnent les femmes. Comme nous l'avons vu précédemment, ce sont les communistes qui, pour pouvoir «libérer» les femmes, les ont considérées comme des non-citoyennes économiques, niant leur travail, tant dans la maison qu'en dehors. La propagande communiste sur le statut des femmes chinoises a ainsi profondément biaisé leur image sociale. Cette manipulation idéologique mériterait donc d'être mieux prise en compte dans cette discussion.

Le chapitre IX offre des études de cas passionnantes sur des femmes de Pékin, cependant l'analyse de l'auteure pêche par une contradiction latente: pourquoi les femmes décrites, qui sont très indépendantes, auraient-elles intérêt à soutenir un patriarcat qui les opprimerait? Au-delà des raisons mentionnées, "pragmatiques, idéologiques et éthiques", il est possible d'interroger la nature du lien entre les discours valorisant les hommes et les pratiques établissant l'indépendance des femmes, un lien qui fonde la distinction de sexe et qui entre en contradiction avec la thèse de l'égalité supposée idéale défendue dans le livre. La question sous-jacente est de savoir s'il faudrait être égaux pour ne pas être patriarcaux?

Pour appréhender les contributions différenciées des deux sexes et cesser de voir le travail féminin, domestique ou autre, comme forcément négligeable ou secondaire, pour satisfaire l'appétit symbolique de hiérarchie des Chinois, il me semble qu'une analyse en termes de complémentarité et de distinction serait plus judicieuse. Concevoir les rapports entre les sexes uniquement comme des rapports de domination et d'opposition limite leur compréhension, alors que les liens d'affection et d'entraide sont aussi à prendre en compte. À ce titre, les auteurs du livre emploient très peu la notion de "sexe», utilisant prioritairement celle de "genre», désormais passée dans le vocabulaire anthropologique, mais signant ainsi une vision moderne, voire postmoderne, et conflictuelle des rapports hommes-femmes partout dans le monde.

\section{Catherine Capdeville-Zeng}




\section{L'évolution des pratiques}

Les différent chapitres de l'ouvrage aboutissent à deux constats opposés, celui d'un "soutien au patriarcat" et celui d'une tendance vers une société plus individualiste où les distinctions de sexe et de génération se sont transformées. Les tenants de la première tendance sont majoritaires (huit contributions sur douze), mais tout en montrant aussi que le patriarcat actuel a fait l'objet d'une «reconfiguration" par rapport à sa forme classique (ou du moins supposée telle). Cette reconfiguration va dans le sens d'un affaiblissement ou d'un adoucissement des normes coercitives, et vers le fait que les femmes prennent part activement à la préservation de ce modèle.

On est donc dans une situation complexe où les informations divergent sur les nouvelles orientations de la société chinoise, qui semble osciller entre une reconfiguration du patriarcat classique - soit une évolution minime - et une tendance allant vers un individualisme plus prononcé dans les organisations familiales - soit une évolution d'envergure. C'est ce que soulignait déjà l'anthropologue sino-américain Yan Yunxiang en 2009, à propos du «paysage moral» chinois:

«La société chinoise est en train de connaître présentement une transition rapide mais sans une direction claire [...]. Nous avons maintenant besoin de plus d'études approfondies qui aideront à mieux comprendre les divers aspects du paysage moral changeant en Chine» (2009b: 23).

Les conclusions de Andrew B. Kipnis (chap. V) sont, à ce titre, très intéressantes, puisqu'elles font apparaître que la modernisation ne doit pas être comprise comme un phénomène uniforme et que les personnes sont impactées différemment selon leur statut social, âge, sexe, lieu d'origine, lieu de travail (etc.), démontrant ainsi que les relations de parenté représentent un cadre flexible adaptable selon les contextes. Envisager un patriarcat unilatéral sans tenir compte des diversités des situations est donc incohérent et met en question l'amalgame entre patrilinéarité et patriarcat.

Si le côté maternel semble peser davantage de nos jours, la tendance de la société urbaine est aussi d'aboutir à un mariage plus égalitaire, tel que dépeint par William Jankowiak et Xuan Li (chap. VII), où la famille étendue laisse peu à peu la place à une famille conjugale et où les conjoints sont plus des agents individuels porteurs de leurs propres désirs que des acteurs de rôles sociaux. Cette analyse expose clairement les effets de l'individualisation chinoise en cours, au-delà des résistances et des détours que suppose cette évolution sociale; elle est également celle qui mesure le mieux le degré d'abandon du patriarcat présumé d'origine. 
L'enquête sur le faux mariage cérémoniel de lesbiennes et de gays (chap. VIII) soulève le problème de l'importance de la norme familiale par rapport à l'acceptation nouvelle de l'homosexualité. On ne comprend notamment pas bien en quoi des lesbiennes, en tant que femmes, seraient si enclines à préserver un ordre patriarcal discriminant. Leur souhait est de ne pas apparaître socialement déviantes, pourtant, dans l'intimité des familles, leur orientation sexuelle est parfois acceptée comme le confirment les expressions inventées pour nommer les partenaires féminines. Le faux mariage se conjugue en effet avec une vie homosexuelle souvent réalisée, ce qui représente un grand pas en avant au niveau social global. Si la façade cérémonielle est de l'ordre du discours - et si ce discours est loin de vouloir retranscrire des "vérités" comme le montre brillamment l'exemple donné dans ce texte, où l'on ne craint pas de réaliser une fausse cérémonie pour ménager l'opinion publique et où l'on ne craint pas non plus de mentir à ses parents pour «ne pas leur faire de la peine» (p. 167) - ne négligeons pas pour autant la modernité de l'avancée sous-jacente, à savoir que, en Chine aussi, des vies de couples homosexuels deviennent dorénavant possibles dans certains contextes urbains, sous couvert donc de divers masques de normalité en l'absence de reconnaissance du mariage homosexuel. Dans ce cadre, le paramètre de l'affection envers les parents - procédant de la «relation humaine» (renqing 人情) - semble essentiel pour comprendre les relations contemporaines.

Le chapitre sur les grands-mères paternelles qui élèvent leurs petits-enfants (chap. IV) se concentre sur la bien lourde tâche qui leur incombe, puisque ces grands-mères ne sont plus aidées par leurs belles-filles, éloignées de leurs enfants à cause des migrations économiques. Mais, on pourrait aussi se demander ce que cela implique pour ces enfants séparés de leurs propres parents et, plus largement, ce que cela révèle, malgré l'entraide pérenne, de l'isolement de plus en plus notable de certains des acteurs sociaux dans la société chinoise contemporaine.

L'enquête sur les dons de sperme (chap. XI) atteste que la Chine aussi s'inscrit désormais dans une mentalité moderne valorisant le biologique au détriment du social. L'adoption ayant toujours été largement pratiquée en Chine pour pallier le manque d'enfants, on aurait pu penser que le don de sperme ne poserait pas trop de problèmes, or c'est le contraire qui se produit. Sans doute cette évolution est-elle parallèle à la montée de l'individualisation qui, en mettant l'accent sur la personne, rend suspicieuse la mise en commun de caractères aussi éminemment personnels que le sperme. Cette enquête tend donc à prouver plus irréfutablement que les autres l'existence d'une survalorisation des hommes, le paradoxe étant que le don de sperme est une pratique récente et non ancienne. Ajoutons que ce poids sur le biologique accompagne l'idéologie nationaliste prônée par

\section{Catherine Capdeville-Zeng}


le gouvernement. Ainsi, l'accès à la modernité ne se fait pas de manière uniforme selon les domaines considérés et l'évolution notable est bien celle de la valorisation de l'individu.

En abordant le nouvel équilibre qui caractérise aujourd'hui les relations entre parents et enfants et entre enfants de sexe opposé (chap. XII), Hong Zhang décrit la tendance allant vers une individualisation des parents âgés qui, après avoir entretenu enfants et petits-enfants, cherchent à vivre de façon beaucoup plus indépendante. L'auteur note aussi le désarroi des familles qui n'ont pas les moyens de faire face financièrement aux coûts élevés de l'industrie naissante des soins aux plus âgés, d'où il résulte que l'inégalité sociale qui en découle se substitue à l'inégalité intra-familiale précédente.

Finalement, cet ouvrage révèle une image assez dynamique de l'organisation familiale chinoise, capable de se renouveler pour s'adapter aux problématiques modernes, tout en conservant des structures d'autorité où les hommes sont toujours en position de supériorité, cependant que les femmes, les filles et belles-filles ont vu leurs situations revalorisées dans bien des domaines (mais elles restent globalement exclues des postes de gouvernance). Ainsi, le mariage devient plus égalitaire quand les hommes ont le désir nouveau d'être aimés par et d'aimer leur femme. Surtout, la famille chinoise contemporaine apparaît dans ce livre comme un lieu de relations communautaires, d'entraide et d'affection.

Dans le contexte de transformation actuel, il est inévitable que l'évolution sociale ne soit pas uniforme et monolithique, ni strictement linéaire. Il est aussi vraisemblable que la Chine crée une forme d'individualisme propre à son expérience historique particulière, forcément différente des formes occidentales. À la question de savoir si la Chine est maintenant entrée dans l'ère de l'individualisme, les tenants des deux orientations de ce livre montrent tous qu'il existe une tendance de plus en plus marquée en ce sens, et divergent finalement seulement sur le degré et l'intensité de ce processus, ainsi que sur l'interprétation que l'on peut en faire. Si les valeurs familiales et l'interdépendance restent fondamentales, elles semblent composer avec une autre nette évolution de la société en termes d'autonomisation et de distanciation. Cet équilibre parfois difficile est évidemment porteur de tensions, qui accentuent en retour la réponse autoritaire du gouvernement.

Ainsi, les paradoxes signalés dans l'introduction sont seulement les signes d'une reconfiguration des valeurs, où celle accordée à l'individu autonome augmente, quoiqu'en restant toujours bien en deçà de ce qu'elle est ailleurs, notamment en Occident. La valeur de l'accent porté à la communauté fait place à une «individualisation " croissante ${ }^{11}$, mais sans doute pas encore

11. Pour reprendre le titre de l'ouvrage de Yan Yunxiang (2009a). 
dominante. Ce système est également révélateur d'une hiérarchie de valeurs marquée par des tendances contraires, avec des inversions opérant à certains niveaux subordonnés. Par exemple, le discours sur l'aide des femmes, qui est en contradiction avec la place essentielle qu'elles occupent sur le plan économique, manifeste la résistance de la société à l'égalisation des statuts en réaffirmant la supériorité de celui des hommes, tout en l'atténuant de façon importante pour donner la possibilité à celui des femmes d'être amélioré. Il faudrait donc sortir de la lecture unilatérale de Max Weber pour s'intéresser à d'autres courants en anthropologie, afin de mieux comprendre la société chinoise et envisager que ses "paradoxes" forment un système.

Comme contre-exemple aux enquêtes de l'ouvrage, voici une anecdote tirée de mes propres observations ethnographiques: dans les années 2000, alors que je résidais dans un village chinois pour y étudier son théâtre local au moment du Nouvel An, se présenta un haut cadre de la province, venu participer à la fête. Aussitôt arrivé, il demanda à rencontrer la mère du chef du village et non ce dernier, ni même son père. Il l'a longuement congratulée pour son travail et ses réalisations, puis pour celles de son fils et des habitants du village. À cette occasion, j'ai donc pu clairement constater que la hiérarchie entre les sexes n'est pas une règle statique, mais qu'elle inclut des lieux et des temps d'inversion, tel celui-ci. Sans doute s'agit-il de la performance d'une parole rituelle, son intérêt étant qu'elle contredit celle des femmes qui «aident» rapportée dans l'ouvrage et qu'elle est proférée par un homme de pouvoir. Le jeu social chinois renvoie ainsi à un espace complexe où les hiérarchies sont manipulées, reformulées, renversées, mettant en défaut la notion de patriarcat appliquée uniformément et de façon immuable à cette société. En survalorisant les hommes et en niant la nécessaire complétude entre les sexes, cette notion omet de prendre en compte les retournements possibles, les alliances éphémères ou pérennes et, finalement, elle réduit les femmes au rôle de victimes, soit consentantes, soit incapables d'agir en tant qu'êtres humains dignes. Elle ne saisit pas non plus la séparation existant entre les pratiques et les discours, alors que les seconds sont souvent là pour travestir les premières lorsqu'elles sont contraires à l'orthodoxie. Une anthropologie plus subtile marquerait la distance par rapport à ces discours affichés et tenterait de les comprendre en rapport ou en opposition aux pratiques et en faisant la part des inversions toujours possibles. Sans doute, dans le cas précis du chapitre sur l'aide des femmes, la méthode d'enquête quantitative est-elle partiellement responsable de ce décalage, car elle privilégie le discours direct sans prendre la pleine mesure des contextes globaux. 
De plus, il faudrait aussi se distancier des assertions courantes, de même que des présupposés propres à une certaine anthropologie, ce qui permettrait de relativiser certains faits bruts, comme la soi-disant prédominance, autrefois en Chine, de la famille étendue, mentionnée par plusieurs contributeurs. Comme l'ont pourtant montré, entre autres, Maurice Freedman (1965) et Hugh Baker (1979), la famille chinoise étendue était avant tout un idéal mais, dans la réalité des pratiques, la famille chinoise était le plus souvent de taille réduite. Ce fait numérique indique que le poids du lignage patrilinéaire au niveau familial a été surestimé, et qu'il l'est encore.

Si l'on s'intéresse maintenant aux relations avec les affins et les maternels signalées dans plusieurs contributions, elles suscitent semble-t-il un certain étonnement chez leurs auteurs, car leur importance est contradictoire avec les postulats inhérents à la notion de patriarcat. Or, beaucoup de sources, anciennes comme contemporaines, mentionnent une relation aux affins très marquée en Chine, certes variable selon les régions. Comme cela n'entre pas dans le schéma du patriarcat, ni dans celui des penseurs (Morgan, Engels, Weber...) ayant élaboré cette notion, qui dans sa modalité patrilinéaire doit l'exclure ou fortement la minimiser, cette forme de relation a corrélativement été sous-évaluée voire évacuée par les anthropologues. Il faut l'honnêteté de Charles Stafford, dans son article précité «Actually Existing Chinese Matriarchy" (2009), pour décrire le malaise qu'il a ressenti à son arrivée à Taiwan lorsqu'il a découvert une situation des femmes différente de tout ce qu'il en avait appris auparavant à l'université anglaise. Rappelons qu'il est dangereux de faire un amalgame entre le système patrilinéaire et les relations entre les sexes, comme cela est évoqué dans la phrase suivante: «l'idéologie patrilinéaire locale est encore assez forte pour entraîner le système entier dans la direction patrilinéaire, suggérant que les relations de genre changent moins vite que les relations de générations» (p. 108). La patrilinéarité représente une certaine idée de la filiation, tandis que les relations entre les sexes se placent sur un autre plan. Il est fallacieux également d'associer une forme de filiation directement à un modèle de société: les sociétés matrilinéaires prouvent bien que ce lien ne peut être forcément direct, puisque, chez elles aussi, les hommes sont encore globalement en position de supériorité (cf. Lévi-Strauss 1949). D'autant que cela voudrait dire, en poussant un peu la logique de cette analyse, que toutes les sociétés sont patriarcales. S'il en est ainsi, quel intérêt y a-t-il alors à utiliser ce terme? Une lecture plus nuancée du système patrilinéaire chinois devrait être faite, en suivant par exemple les analyses de Marcel Granet. Or, à ma connaissance, peu d'enquêtes anthropologiques d'envergure se sont vraiment intéressées aux relations d'affinité en Chine. Si le caractère patrilinéaire chinois ne fait pas de doute, les indices portant sur les aspects bilatéraux des relations familiales devraient être mieux évalués. 
Une réflexion sur l'organisation familiale chinoise devrait ainsi s'écarter de la vision occidentalisée ou wébérienne d'un rapport de domination entre les sexes et les générations, et privilégier une étude sur la complémentarité des statuts sociaux, de sexes, d'âges, de générations, de situations politiques et géographiques (etc.), tout en mettant en jeu la profondeur relative des relations humaines. Si l'on s'attache à étudier comment une société d'essence hiérarchique - une société holiste - s'organise pour devenir une société individualiste, il faut encore relativiser le message confucianiste et comparer les faits et les discours pour ne pas tomber dans les travers des penseurs réformateurs puis communistes des années républicaines, rapportés ci-dessus.

La façon dont la Chine des Han a mis en place une société configurée par un système patrilinéaire associé à une idéologie prônant la hiérarchie sociale doit aussi prendre en compte le fait que le confucianisme a été et est toujours tempéré par d'autres courants, notamment le taoïsme et le bouddhisme, et qu'il s'accompagne de pratiques sociales contradictoires ou complémentaires, comme l'héritage équitable entre les frères, l'équivalence des descendants devant leurs ancêtres, les systèmes de rotation pour les charges afférentes aux temples, pratiques auxquelles on peut associer la place importante des femmes dans la famille, leur gestion de leur cassette personnelle, etc. C'est bien ce système complexe pour lequel, à une hiérarchie présentée ouvertement et de façon englobante, s'ajoutent des inversions de statuts, notamment dans les positions subordonnées, qui parait incompréhensible aux auteurs de ce livre, sous prétexte qu'il est empli de "paradoxes» (p. 7). En effet, l'adjonction du terme patriarcat à la famille chinoise occulte cette situation contrastée et empêche d'en saisir le fonctionnement. Ainsi les inversions sont comprises comme des "points faibles", alors qu'elles sont inhérentes à toute société, les organisations sociales étant toujours complexes et ne s'orientant jamais unilatéralement dans une seule direction.

Si la famille joue toujours un rôle essentiel, cela n'est pas antagoniste avec l'orientation générale de la société, c'est parce qu'elle présente une configuration holiste qui adoucit la violence des relations sociales propre à un système social effectivement de plus en plus individualiste. Selon Louis Dumont (1983: 28), dans la société occidentale aussi la famille atteste d'une survivance d'éléments prémodernes, qui participe de sa structure sociale contemporaine combinant une configuration individualiste avec la présence de reliquats d'holisme.

Ajoutons, enfin, que malgré cette tendance vers plus d'individualisme, la relation prioritaire actuelle dans la famille reste l'interdépendance, qui n'implique pas automatiquement une "domination " unilatérale des pères et des hommes, mais la mise en pratique d'une "distinction de sexe» et d'une "distinction de génération», où hommes et femmes et parents et enfants 
coopèrent sur un mode hiérarchisé. Ainsi, la famille chinoise s'oppose à l'État omniprésent, comme son antithèse, et comme un espace où des relations de type holiste continuent de prévaloir. S'il y a un totalitarisme en Chine, il n'est pas à chercher prioritairement dans les relations familiales ou dans un supposé patriarcat. Comme Claude Lévi-Strauss, je pense qu'il se situe plutôt dans le domaine politique et qu'il procède du legs du "légisme» (fajia 法家), idéologie autoritaire de la première dynastie impériale des Qin (221-207 av. J.-C.), dont la Chine n’a jamais vraiment réussi à se défaire (Vandermeersch 1965; Schiele 2017; Capdeville-Zeng 2017).

Institut national des langues et civilisations orientales Département d'études chinoises (IFRAE/CECMC), Paris catherine.capdeville@inalco.fr

MOTS CLÉS/KEYWORDS: patriarcat/patriarchy - distinction de sexe/sex distinction - distinction de génération/generation distinction - relations familiales/family relations - piété filiale/filial piety - système patrilinéaire/patrilineal system - système hiérarchique/hierarchical system individualisme/individualism - Chine/China.

\section{RÉFÉRENCES CITÉES}

Bachofen, Johan Jakob

1996 [1861] Le Droit maternel. Recherche sur la gynécocratie de l'Antiquité dans sa nature religieuse et juridique. Trad. de l'allemand et préface par Étienne Barilier. Lausanne, L'Âge d'homme.

Baker, Hugh D. R.

1979 Chinese Family and Kinship.

New York, Columbia University Press.

Billeter, Jean-François

1991 "La civilisation chinoise», in Jean Poirier, ed., Histoire des mours,

2. Modes et Modèles. Paris, Gallimard («Encyclopédie de la Pléiade» 48) : 865-931.

Bonte, Pierre \& Michel Izard, eds 2002 [1991] Dictionnaire de l'ethnologie et de l'anthropologie. Paris, Presses universitaires de France ("Quadrige»).

\section{Capdeville-Zeng, Catherine}

2017 «Groupes rock contemporains et idéologies chinoises: pouvoir impérial, autorité confucianiste, non-pouvoir taoïste", in David Gibeault \& Stéphane Vibert, eds, Autorité et pouvoir en perspective comparative. Paris, Presses de l'Inalco ("TransaireS»): 327-357. En ligne: https://books.openedition.org/pressesinalco/2368

\section{Cohen, Myron L.}

2005 Kinship, Contract, Community, and State. Anthropological Perspectives on China. Stanford, Stanford University Press ( Studies of the Weatherhead East Asian Institute»).

Davis, Deborah S. \& Sara K. Friedman, eds 2014 Wives, Husbands and Lovers. Marriage and Sexuality in Hong Kong, Taiwan and Urban China. Stanford, Stanford University Press. 
Doeblin, Alfred, ed.

1947 Les Pages immortelles de Confucius.

\section{Dumont, Louis}

1983 Essais sur l'individualisme.

Une perspective anthropologique sur l'idéologie

moderne. Paris, Le Seuil («Esprit»).

\section{Engels, Friedrich}

1884 Der Unsprung der Familie, des Privateigenthums und des Staats L = L'Origine de la famille, de la propriété privée et de l'État]. Hottingen-Zürich, Verlag der Schweizerischen Volksbuchhandlung.

Fei Xiaotong (费孝通)

1992 [1945] From the Soil. The Foundations of Chinese Society. A translation of Fei Xiaotong's Xiangtu Zhongguo, with an introduction and epilogue by Gary G. Hamilton and Wang Zheng. Berkeley, University of California Press.

Fourier, Charles

1830 Le Nouveau Monde industriel et sociétaire, ou Invention du procédé d'industrie attrayante et naturelle, distribuée en séries passionnées. Paris, Bossange Père.

\section{Freedman, Maurice}

1965 Lineage Organization in Southeastern China. London, University of LondonAthlone Press / New York, Humanities Press («Monographs on Social Anthropology» 18).

\section{Granet, Marcel}

1939 Catégories matrimoniales et relations de proximité dans la Chine ancienne. Paris, Félix Alcan («Bibliothèque de philosophie contemporaine»).

1968 [1934] La Pensée chinoise. Paris, Albin Michel («L'Évolution de l'humanité» 3).

Héran, François

2009 Figures de la parenté.

Une histoire critique de la raison

structurale. Paris, Presses universitaires

de France («Sociologies»).

\section{Hershatter, Gail}

2003 "Making the Visible Invisible:

The Fate of "The Private" in Revolutionnary China", in Lü Fangshang, ed., Wu sheng zhi sheng, 1. Jin dai Zhongguo de fu nü yu guo jia (1600-1950) [= Voices Amid Silence, 1. Women and the Nation in Modern China (1600-1950)]. Taiwan, Institute of Modern History-Academia Sinica: 257-281.

\section{Kandiyoti, Deniz}

1988 "Bargaining with Patriarchy", Gender and Society 2 (3): 274-290.

\section{Lévi-Strauss, Claude}

1949 Les Structures élémentaires de la parenté. Paris, Presses universitaires de France («Bibliothèque de philosophie contemporaine»).

\section{Li Xia (李霞)}

2010 Natal Family and Married-in Family. Women's Living Space and Backstage Power in a North China Village (娘家与婆家一华 北农村妇女的生活空间和后台权力 Niangjia yu pojia. Huabei nongcun funü de shenghuo kongjian he houtai quanli). Beijing, Social Sciences Academic Press.

Mencius

2003 Mencius. Traduit du chinois par André Lévy. Paris, You-Feng.

\section{Morgan, Lewis Henry}

1877 Ancient Society, or Researches in the Line of Human Progress from Savagery, through Barbarism to Civilization.

New York, H. Holt \& Company.

\section{Schiele, Alexandre}

2017 La Chine postmaoïste: un État légiste au XXe siècle. Analyse sociohistorique et analyse des discours de Deng Xiaoping (1975-1992). Montréal, Université du Québec à Montréal, thèse de doctorat.

\section{Stafford, Charles}

2009 «Actually Existing Chinese Matriarchy", in Suzanne Brandstädter \& Gonçalo D. Santos, eds, Chinese Kinship. Contemporary Anthropological Perspectives. London-New York, Routledge: 137-153. 


\section{Vandermeersch, Léon}

1965 La Formation du légisme. Recherche sur la constitution d'une philosophie politique caractéristique de la Chine ancienne. Paris, Publ. de l'École française d'Extrême-Orient.

1991 «Le mariage suivant le rituel confucianiste", in Yuzô Mizoguchi \& Léon Vandermeersch, eds, Confucianisme et sociétés asiatiques. Paris, L'Harmattan

(«Recherches asiatiques»): 53-68.

\section{Wang Mingming (王铭铭)}

2009 Empire and Local Worlds.

A Chinese Model for Long-Term Historical

Anthropology. Walnut Creek, Left Coast

Press («Publications of the Institute of Archaeology, University College London, Critical Perspectives on Cultural Heritage»).

2014 The West as the Other. A Genealogy of Chinese Occidentalism. Hong Kong, Chinese University Press.

Weber, Max

1978 [1921] Economy and Society. An Outline of Interpretive Sociology.

Ed. by Guenther Roth and Claus Wittich.

Transl. by Ephraim Fischoff et al.

Berkeley, University of California Press.
Wolf, Margery

1972 Women and the Family in Rural

Taiwan. Stanford, Stanford University Press.

Yan Yunxiang

1997 «The Triumph of Conjugality: Structural Transformation of Family Relations in a Chinese Village ", Ethnology 36 (3) : 191-212.

2009a The Individualization of Chinese Society. Oxford-New York, Berg («London School of Economics Monographs on Social Anthropology» 77).

2009b «The Good Samaritan's New Trouble: A Study of the Changing Moral Landscape in Contemporary China", Social Anthropology 17 (1): 9-24.

\section{Zufferey, Nicolas}

2003 « La condition féminine traditionnelle en Chine: état de la recherche", Études chinoises 22: 185-229. 